\title{
Performance Enhanced Robust Iterative Learning Control with Experimental Application to PMSM Position Tracking
}

\author{
Slawomir Mandra, Krzysztof Galkowski \\ Institute of Physics, Faculty of Physics, Astronomy and Informatics, \\ Nicolaus Copernicus University, \\ Grudziadzka 5, 87-100 Torun, Poland and \\ Institute of Control and Computation Engineering, \\ University of Zielona Gora, \\ Szafrana 2, 65-516 Zielona Gora, Poland. \\ Eric Rogers \\ Department of Electronics and Computer Science \\ University of Southampton, Southampton SO17 1BJ, UK. \\ (etar@ecs.soton.ac.uk) \\ Andreas Rauh, Harald Aschemann, \\ Chair of Mechatronics, \\ University of Rostock, \\ Justus-von-Liebig-Weg 6, D-18059 Rostock, Germany.
}

\begin{abstract}
This paper develops an innovative robust iterative learning control law using the repetitive process setting. The new design is experimentally validated through a comprehensive set of experiments highlighting the capabilities for position tracking control of a permanent magnet synchronous motor subject to load disturbances in the presence of uncertainties in selected parameters.
\end{abstract}

\section{INTRODUCTION}

Iterative learning control (ILC) was especially developed for systems or processes that repeat the same finite duration operation over and over again, such as gantry robots in mass production facilities. Each repetition is termed a trial 
and its duration the trial length. Once a trial is completed, all information generated are available for updating the control input so that the output follows the specified reference trajectory, including the cases where there is uncertainty in the plant model and/or load disturbances are present. The notation used in this paper is of the form $h_{k}(p), 0 \leq p \leq \alpha-1$, where $h$ is a scalar or vectorvalued function under consideration, the nonnegative integer $k$ denotes the trial number and $\alpha<\infty$ the number of samples along the trial in the discrete case ( $\alpha$ times the constant sampling period gives the trial length).

Let $y^{\text {ref }}(p)$ denote the supplied reference trajectory. Then, the error on trial $k$ is $e_{k}(p)=y^{\text {ref }}(p)-y_{k}(p)$ where $y_{k}(p)$ is the output on trial $k$ and the objective is the design of a controller to ensure that $\left\{e_{k}\right\}_{k}$ converges to zero in $k$ and the control input signals to a learned control. The first work on ILC is widely credited to [1] and since then it has been an expanding area of research in control systems with an ever widening range of applications. Starting points for the literature are the survey papers $[2,3]$.

In the case of discrete linear dynamics, there are many settings for ILC design. In this paper, the repetitive process setting is used, which has already seen many designs reported, e.g., [4] with experimental validation. However, repetitive process based designs come at a price in terms of data storage required that may be problematic in some applications. This paper develops a new design where storage of the previous trial state vector over the complete trial length is not required and also introduces compensation for other unwanted effects.

The new contributions in this paper are: i) integral action to compensate for unknown constant or slowly varying disturbances on the trial, where the control action is applied on the trial in which they first appear, rather than on subsequent trials in other designs, ii) feedforward control action to reduce initial tracking errors in the early learning phase and iii) allows for plants with relative degree greater than unity unlike the alternative design in [5].

Model uncertainty described by the convex polytope is include in the design. Moreover, the merits of the new design are illustrated by applying the control strategy to a Permanent Magnet Synchronous Motor (PMSM). Finally, a comparison in application terms with competing repetitive process based ILC designs is given.

Throughout this paper, the zero and identity matrices with compatible dimensions are denoted by $\mathbf{0}$ and $\mathbf{I}$, respectively. A real symmetric positive (negative) definite matrix is written as $\mathbf{Z} \succ 0(\prec 0)$ and $\mathbf{X} \preceq \mathbf{Y}$ denotes the case when $\mathbf{X}-\mathbf{Y}$ is a symmetric negative semi-definite matrix. The symbol $(\star)$ denotes block entries in a symmetric matrix. 


\section{Control Configuration}

The new ILC design in this paper is based on a discrete-time linear parameterdependent state-space plant model written in the ILC setting as

$$
\begin{aligned}
x_{k+1}(p+1)= & \mathbf{A}(\lambda) x_{k+1}(p) \\
& +\mathbf{B}(\lambda)\left(u_{k+1}(p)-d_{k+1}(p)\right), \\
y_{k+1}(p)= & \mathbf{C} x_{k+1}(p),
\end{aligned}
$$

where the matrices $\mathbf{A}(\lambda)$ and $\mathbf{B}(\lambda)$ are assumed to belong to a convex polytope described by

$$
\begin{aligned}
& {\left[\begin{array}{ll}
\mathbf{A}(\lambda) & \mathbf{B}(\lambda)
\end{array}\right]=\sum_{j=1}^{M} \lambda_{j}\left[\begin{array}{ll}
\mathbf{A}_{j} & \mathbf{B}_{j}
\end{array}\right]} \\
& \lambda=\left[\begin{array}{c}
\lambda_{1} \\
\vdots \\
\lambda_{M}
\end{array}\right], \quad \lambda_{j} \geq 0, \quad \sum_{j=1}^{M} \lambda_{j}=1 .
\end{aligned}
$$

Considering trial $k+1, x_{k+1}(p) \in \mathbb{R}^{n}$ denotes the state vector, $u_{k+1}(p) \in \mathbb{R}$ the control input, $d_{k+1}(p) \in \mathbb{R}$ the load disturbance input and $y_{k+1}(p) \in \mathbb{R}$ the output. The output vector $\mathbf{C}$ is assumed to be fixed and known exactly and the relative degree $r \geq 1$. This paper considers single-input single-output (SISO) systems but the results easily generalize to multi-input multi-output (MIMO) systems.

In the repetitive process setting, the ILC law is of the form

$$
\begin{aligned}
u_{k+1}(p)= & u_{k}(p)+\mathbf{K}_{\text {stab }}\left(x_{k+1}(p)-x_{k}(p)\right) \\
& +K_{\mathrm{ILC}} e_{k}(p+1)
\end{aligned}
$$

where the second term provides stabilizing control action and the third term is the ILC feedforward using sample $p+1$ of the error information from the previous trial to improve tracking accuracy during the current trial. The design of this control law is based on linear repetitive process stability theory, and the associated control law gain matrices are computed using linear matrix inequalities (LMIs). The new ILC scheme developed in this paper is shown in Fig. 1 and adds the following features relative to previous designs.

1. The tracking error for a system subject to a load disturbance is reduced by existing ILC laws but starting from the next trial. In the new design the impact of such a disturbance is compensated for on the trial where it appears first and hence a possibly significant tracking error is avoided.

2. Large tracking errors occurring in the early trials or directly after a change in the reference trajectory are reduced.

3. Design for plants with relative degree $r \geq 1$ is allowed.

4. ILC algorithm implementation does not require additional memory to store $\mathbf{K}_{\text {stab }} x_{k}(p)$ in (3). 


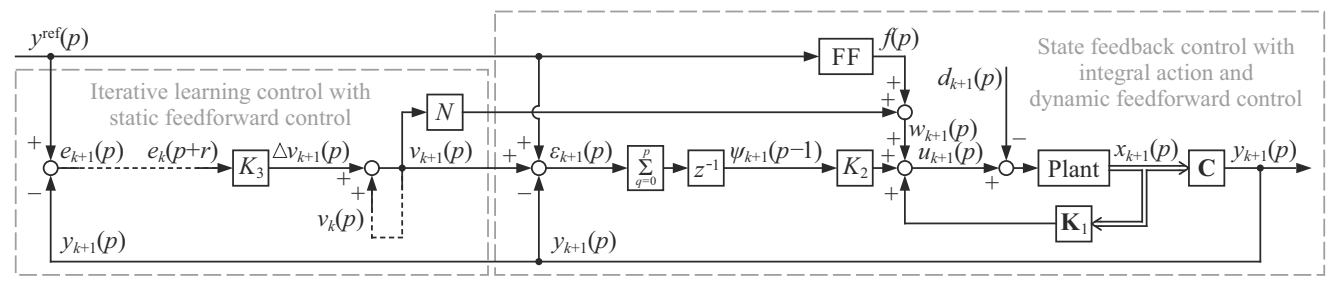

Figure 1: Block diagram of the ILC structure based on a state feedback controller with integral action and a dynamic feedforward controller.

The term $d_{k+1}$ in Fig. 1 represents a load disturbance entering on trial $k+1$, resulting in a steady-state tracking error in the along the trial dynamics (the response along a trial are described in terms of the discrete-time variable $p$ ). This error can be reduced by the ILC law (3) but this compensation will only take effect on the next trial after the disturbances have occurred. One suitable countermeasure was given in [5], where the tracking error caused by disturbances is reduced by a disturbance observer. The stability of such an observer-based ILC can be proven after the design is complete, see [5]. This is not the case with the new design in this paper.

Another way of immediately reducing the tracking error caused by unknown constant or slowly varying disturbances, within the trial where they first appear, is to include integral action of the current tracking error. The implementation of this action is achieved by the output of the $K_{2}$ block in Fig. 1. Moreover, a disturbance observer also cannot remove this error completely due to limited dynamics but instead it is reduced to zero by ILC action on subsequent trials. To increase the convergence speed, the ILC signal $v_{k+1}(p)$ acts on the closedloop (state feedback) system by both the static feedforward gain $N$ and the integral action.

In an ILC implementation, large tracking errors can arise during early trials or directly after a change in the reference trajectory, see [5]. One cause of errors in the initial trials is the assumption that the initial control signal is zero $\left(u_{0}(p)=0\right)$. Errors after a change in the reference trajectory may arise, among other reasons, from the fact that stored information on the previous control input $\left(u_{k}(p)\right)$ is no longer valid after this change and may even prevent accurate tracking. The control design developed in this paper aims at compensating this effect by including dynamic feedforward control. It takes advantage of the reference trajectory $y^{\text {ref }}(p)$ and is implemented within the FF block in Fig. 1 and the ILC update is given by $K_{3} e_{k}(p+r)$, building on previous analysis in, e.g., [4]. 


\section{Robust state feedback with integral action and feedforward control design}

The ILC scheme of Fig. 1 is based on a state feedback controller with integral action and both static and dynamic feedforward controllers. The design of these controllers is detailed in this section.

The integral action in the state feedback controller is introduced through

$$
\psi_{k+1}(p)=\sum_{q=0}^{p} \varepsilon_{k+1}(q),
$$

where $\varepsilon_{k+1}(p)=y^{\mathrm{ref}}(p)+v_{k+1}(p)-y_{k+1}(p)$. For design purposes, the signals $v_{k+1}(p), y^{\text {ref }}(p)$ and $d_{k+1}(p)$ are set to zero as they do not influence stability. Applying the control law

$$
u_{k+1}(p)=\mathbf{K}_{1} x_{k+1}(p)+K_{2} \psi_{k+1}(p-1)
$$

to the plant model (1) leads to an augmented system representation that can be written as

$$
\begin{aligned}
\chi_{k+1}(p+1) & =\left(\mathbf{A}_{\mathbf{s}}(\lambda)+\mathbf{B}_{\mathbf{s}}(\lambda) \mathbf{K}_{\mathrm{s}}\right) \chi_{k+1}(p), \\
y_{k+1}(p) & =\mathbf{C}_{\mathrm{cl}} \chi_{k+1}(p),
\end{aligned}
$$

where

$$
\begin{aligned}
\chi_{k+1}(p) & =\left[\begin{array}{c}
x_{k+1}(p) \\
\psi_{k+1}(p-1)
\end{array}\right], \\
\mathbf{A}_{\mathbf{s}}(\lambda) & =\left[\begin{array}{cc}
\mathbf{A}(\lambda) & \mathbf{0} \\
-\mathbf{C} & 1
\end{array}\right], \mathbf{B}_{\mathbf{s}}(\lambda)=\left[\begin{array}{c}
\mathbf{B}(\lambda) \\
0
\end{array}\right], \\
\mathbf{C}_{\mathbf{s}} & =\left[\begin{array}{ll}
\mathbf{C} & 0
\end{array}\right], \mathbf{K}_{\mathrm{s}}=\left[\begin{array}{ll}
\mathbf{K}_{1} & K_{2}
\end{array}\right] .
\end{aligned}
$$

The design of the state feedback law with the integral action requires $\mathbf{K}_{\mathrm{s}}$ to be chosen such that (6) is stable for all possible $[\mathbf{A}(\lambda) \mathbf{B}(\lambda)]$. One way of completing this design is to find $u_{k+1}(p)=\mathbf{K}_{\mathbf{s}} \chi_{k+1}(p)$ that minimizes the cost function

$$
J_{\mathrm{cl}}=\sum_{p=0}^{\infty}\left(\chi_{k+1}^{\mathrm{T}}(p) \mathbf{Q} \chi_{k+1}(p)+R u_{k+1}^{2}(p)\right),
$$

where $\mathbf{Q} \succ 0$ and $R>0$ are an appropriately chosen weighting matrix and scalar factors. Many ways of solving this problem are known and in this paper the guaranteed cost method $[6]$ is used.

For given $\chi_{k+1}(0)=\gamma$, suppose that there exist compatibly dimensioned matrices $\mathbf{Y} \succ 0, \mathbf{W}$ and a positive scalar $\beta$ such that for $j=1, \ldots, M$, the LMIs

$$
\left[\begin{array}{cccc}
\mathbf{Y} & (\star) & (\star) & (\star) \\
\mathbf{A}_{\mathrm{s} j} \mathbf{Y}+\mathbf{B}_{\mathrm{s} j} \mathbf{W} & \mathbf{Y} & (\star) & (\star) \\
\mathbf{Y} & \mathbf{0} & \mathbf{Q}^{-1} & (\star) \\
\mathbf{W} & \mathbf{0} & \mathbf{0} & \frac{1}{R}
\end{array}\right] \succeq 0
$$




$$
\left[\begin{array}{ll}
\beta & \gamma^{T} \\
\gamma & \mathbf{Y}
\end{array}\right] \succeq 0
$$

are feasible, where

$$
\mathbf{A}_{\mathbf{s} j}=\left[\begin{array}{cc}
\mathbf{A}_{j} & \mathbf{0} \\
-\mathbf{C} & 1
\end{array}\right], \quad \mathbf{B}_{\mathrm{s} j}=\left[\begin{array}{c}
\mathbf{B}_{j} \\
0
\end{array}\right] .
$$

Then (for the proof see [6]) the following optimization procedure can be applied

$$
\min (\beta), \text { subject to: } \mathbf{Y} \succ 0,(9),(10)
$$

for $j=1, \ldots, M$. If a solution exists to this problem, the stabilizing gain vector is given by

$$
\mathbf{K}_{\mathrm{s}}=\mathbf{W Y}^{-1},
$$

where $\mathbf{K}_{1}$ and $\mathbf{K}_{2}$ are obtained from the partitioning of $\mathbf{K}_{\mathrm{s}}$ according to (7).

The next step is to design the dynamic and static feedforward control terms in Fig. 1. Using the dynamic feedforward controller (FF), accurate tracking can be obtained only if both plant model and the disturbances are exactly known. In the case of an uncertain system model and unknown, but repetitive, disturbances the dynamic feedforward controller only calculates an approximation to the control signal, which is enhanced by the ILC signal $v_{k+1}(p)$ computed using the $K_{3}$ block in Fig. 1. This design can be completed as detailed in Sec. 6 .

In application, the static feedforward controller represented by the block $N$ in Fig. 1 is added to avoid instability arising due to excessive integration. Under the assumption that $K_{2}=0, f(p)=0$ and $d_{k+1}(p)=0$, the resulting closedloop transfer function with the state feedback control law applied has one input, the signal $w_{k+1}(p)=N v_{k+1}(p)$ in Fig. 1. To make the DC gain of the series connection of the block $N$ and the closed-loop transfer function equal to one, the static feedforward gain $N$ has to be chosen as the inverse of the DC gain of the feedback loop.

\section{Repetitive process formulation of the ILC de- sign}

The ILC signal $v_{k+1}(p)$ acts on the closed-loop state feedback control system as shown in Fig. 1. Setting $y^{\text {ref }}(p)=0$ and $d_{k+1}(p)=0$, the contribution of the $v_{k+1}(p)$ signal to the plant output is described by the state-space model

$$
\begin{aligned}
\chi_{k+1}(p+1) & =\mathbf{A}_{\mathrm{cl}}(\lambda) \chi_{k+1}(p)+\mathbf{B}_{\mathrm{ILC}}(\lambda) v_{k+1}(p), \\
y_{k+1}(p) & =\mathbf{C}_{\mathrm{cl}} \chi_{k+1}(p),
\end{aligned}
$$

where

$$
\begin{aligned}
\mathbf{A}_{\mathrm{cl}}(\lambda) & =\mathbf{A}_{\mathrm{s}}(\lambda)+\mathbf{B}_{\mathrm{s}}(\lambda) \mathbf{K}_{\mathrm{s}} \\
\mathbf{B}_{\mathrm{ILC}}(\lambda) & =\left[\begin{array}{c}
\mathbf{B}(\lambda) N \\
1
\end{array}\right]
\end{aligned}
$$


An ILC law computes the signal to be applied on the next trial and often is the sum of the signal used on the previous trial and a correction term, i.e.,

$$
v_{k+1}(p)=v_{k}(p)+\Delta v_{k+1}(p),
$$

where

$$
\Delta v_{k+1}(p)=K_{3} e_{k}(p+r),
$$

in contrast to other repetitive process based designs, e.g. [5].

Introduce, for analysis purposes only, the vector

$$
\eta_{k+1}(p)=\chi_{k+1}(p)-\chi_{k}(p) .
$$

Then, the application of (16) to (14) gives the following state-space model description of the controlled ILC dynamics

$$
\begin{aligned}
\eta_{k+1}(p+1)= & \mathbf{A}_{\mathrm{cl}}(\lambda) \eta_{k+1}(p) \\
& +\mathbf{B}_{\mathrm{ILC}}(\lambda) K_{3} e_{k}(p+r), \\
e_{k+1}(p)= & -\mathbf{C}_{\mathrm{cl}} \eta_{k+1}(p)+e_{k}(p) .
\end{aligned}
$$

By applying the $z$-transform, the following description of the ILC dynamics (19) can be obtained (see, e.g., the relevant references in [3] for the justification of why applying the $z$-transform to the finite trial length does not affect the final result)

$$
\frac{e_{k+1}(z)}{e_{k}(z)}=-\mathbf{C}_{\mathrm{cl}} z^{r}\left(z \mathbf{I}-\mathbf{A}_{\mathrm{cl}}(\lambda)\right)^{-1} \mathbf{B}_{\mathrm{ILC}}(\lambda) K_{3}+1 .
$$

Moreover, see [4]. this leads to the state-space model

$$
\begin{aligned}
\eta_{k+1}(p+1)= & \mathbf{A}_{\mathrm{cl}}(\lambda) \eta_{k+1}(p) \\
& +\mathbf{B}_{\mathrm{ILC}}(\lambda) K_{3} e_{k}(p), \\
e_{k+1}(p)= & -\boldsymbol{\Gamma}(\lambda) \eta_{k+1}(p) \\
& +\left(1-\Upsilon(\lambda) K_{3}\right) e_{k}(p),
\end{aligned}
$$

with

$$
\begin{aligned}
& \boldsymbol{\Gamma}(\lambda)=\mathbf{C}_{\mathrm{cl}}\left(\mathbf{A}_{\mathrm{cl}}(\lambda)\right)^{r}, \\
& \Upsilon(\lambda)=\mathbf{C}_{\mathrm{cl}}\left(\mathbf{A}_{\mathrm{cl}}(\lambda)\right)^{r-1} \mathbf{B}_{\mathrm{ILC}}(\lambda) .
\end{aligned}
$$

The set with vertices $\left[\begin{array}{lllll}\mathbf{A}_{\mathrm{cl} j} & \mathbf{B}_{\mathrm{ILC} j} & \boldsymbol{\Gamma}_{j} & \Upsilon_{j}\end{array}\right]$, where

$$
\begin{gathered}
\mathbf{A}_{\mathrm{cl} j}=\mathbf{A}_{\mathrm{s} j}+\mathbf{B}_{\mathrm{s} j} \mathbf{K}_{\mathrm{s}}, \quad \mathbf{B}_{\mathrm{ILC} j}=\left[\begin{array}{c}
\mathbf{B}_{j} N \\
1
\end{array}\right], \\
\boldsymbol{\Gamma}_{j}=\mathbf{C}_{\mathrm{cl}} \mathbf{A}_{\mathrm{cl} j}^{r}, \quad \Upsilon_{j}=\mathbf{C}_{\mathrm{cl}} \mathbf{A}_{\mathrm{cl} j}^{r-1} \mathbf{B}_{\mathrm{ILC} j}
\end{gathered}
$$

is, in general, non-convex due to the presence of powers of the matrix $\mathbf{A}_{\mathrm{cl}}(\lambda)$. 
There are many tools available to form a convex set given the vertices. Here, MATLAB compatible geometric bounding software is used and gives

$$
\begin{aligned}
& {\left[\begin{array}{llll}
\hat{\mathbf{A}}_{\mathrm{cl}}(\lambda) & \hat{\mathbf{B}}_{\mathrm{ILC}}(\lambda) & \hat{\boldsymbol{\Gamma}}(\lambda) & \hat{\Upsilon}(\lambda)
\end{array}\right]=\tilde{\mathrm{X}}} \\
& \tilde{\mathrm{X}}=\sum_{j=1}^{\mathcal{M}} \lambda_{j}\left[\begin{array}{llll}
\hat{\mathbf{A}}_{\mathrm{cl} j} & \hat{\mathbf{B}}_{\mathrm{ILC} j} & \hat{\boldsymbol{\Gamma}}_{j} & \hat{\Upsilon}_{j}
\end{array}\right]
\end{aligned}
$$

As a result, (21) becomes

$$
\begin{aligned}
\eta_{k+1}(p+1) & =\hat{\mathbf{A}}(\lambda) \eta_{k+1}(p)+\hat{\mathbf{B}}_{0}(\lambda) e_{k}(p), \\
e_{k+1}(p) & =\hat{\mathbf{C}}(\lambda) \eta_{k+1}(p)+\hat{D}_{0}(\lambda) e_{k}(p),
\end{aligned}
$$

with the vertices

$$
\begin{aligned}
\hat{\mathbf{A}}(\lambda)=\hat{\mathbf{A}}_{\mathrm{cl}}(\lambda), & \hat{\mathbf{B}}_{0}(\lambda)=\hat{\mathbf{B}}_{\mathrm{ILC}}(\lambda) K_{3}, \\
\hat{\mathbf{C}}(\lambda)=-\hat{\boldsymbol{\Gamma}}(\lambda), & \hat{D}_{0}(\lambda)=1-\hat{\Upsilon}(\lambda) K_{3}
\end{aligned}
$$

and the set of vertices having the required convexity property. The state-space model (25) is that of a discrete linear repetitive process [7] and the next section uses the stability theory of these processes to undertake robust ILC design.

\section{Robust ILC Design}

A stability theory for linear constant pass length processes has been developed in a Banach space setting [7]. This stability theory requires that a bounded initial pass profile produces a bounded sequence of pass profiles (in $k$ ) either over the finite and fixed pass length or, in stronger form, independent of the pass length. The extension to control law design has been the subject of much research leading to computationally feasible design algorithms. In this paper it is stability along the pass property that is used and to conform with the vast majority of the ILC literature, the word pass is replaced by trial from this point onwards. A standing result is that if stability along the trial holds for a linear repetitive process then ILC dynamics represented in this form have monotonic trial-to-trial error convergence [7].

Introduce the notation

$$
\boldsymbol{\Phi}(\lambda)=\left[\begin{array}{ll}
\hat{\mathbf{A}}(\lambda) & \hat{\mathbf{B}}_{0}(\lambda) \\
\hat{\mathbf{C}}(\lambda) & \hat{D}_{0}(\lambda)
\end{array}\right],
$$

and $\mathcal{P}(\lambda)=\operatorname{diag}\left(\mathbf{P}_{1}(\lambda), P_{2}(\lambda)\right)$. Then by linear repetitive process stability theory $(25)$ is stable along the trial $[7]$ if

$$
\boldsymbol{\Phi}^{\mathrm{T}}(\lambda) \mathcal{P}(\lambda) \boldsymbol{\Phi}(\lambda)-\mathcal{P}(\lambda) \prec 0 .
$$




\subsection{Guaranteed cost function based control design}

The design problem considered in this section is to determine the gain $K_{3}$ that guarantees 'fast' trial-to-trial error convergence. If the LMIs resulting from (28) are feasible, there exist infinitely many solutions but no criteria for selecting them to reflect the expected performance. One way of avoiding this obstacle is to use the guaranteed cost method introduced for standard linear systems in [6] and for repetitive processes in [7]. Since the dynamics along the trial of the ILC system (25) are dependent on (12), the following cost function is used

$$
\begin{aligned}
J_{\mathrm{ILC}} & =\sum_{k=0}^{k^{*}} \sum_{p=0}^{\alpha-1} e_{k+1}^{2}(p) \\
& =\sum_{k=0}^{k^{*}} \sum_{p=0}^{\alpha-1} \xi^{\mathrm{T}}(k, p) \mathbf{U}^{\mathrm{T}}(\lambda) \mathbf{U}(\lambda) \xi(k, p),
\end{aligned}
$$

with $\mathbf{U}(\lambda)=\left[\begin{array}{ll}\hat{\mathbf{C}}(\lambda) & \hat{D}_{0}(\lambda)\end{array}\right]$ and the number $k^{*}$ of trials to be completed. Hence the requirement for stability along the trial becomes

$$
\boldsymbol{\Phi}^{\mathrm{T}}(\lambda) \mathcal{P}(\lambda) \boldsymbol{\Phi}(\lambda)-\mathcal{P}(\lambda)+\mathbf{U}^{\mathrm{T}}(\lambda) \mathbf{U}(\lambda) \preceq 0 .
$$

The following is the main result of this paper.

Theorem 1 Consider uncertain discrete ILC dynamics described by (25). Then the robust stable along the trial property holds and the associated cost function $J_{\text {ILC }}(29)$ satisfies the upper bound in Theorem 2 of [5] if, for given boundary conditions,

$$
\eta_{k+1}(0)=\mathbf{f}, \quad e_{0}(p)=g
$$

the LMIs

$$
\left[\begin{array}{ccccc}
\mathbf{G}_{1}+\mathbf{G}_{1}^{\mathrm{T}}-\mathbf{Y}_{1 j} & (\star) & (\star) & (\star) & (\star) \\
\mathbf{0} & 2 G_{2}-Y_{2 j} & (\star) & (\star) & (\star) \\
\hat{\mathbf{A}}_{\mathrm{clj}} \mathbf{G}_{1} & \hat{\mathbf{B}}_{\mathrm{ILC} j} W & \mathbf{Y}_{1 j} & (\star) & (\star) \\
-\hat{\boldsymbol{\Gamma}}_{j} \mathbf{G}_{1} & G_{2}-\hat{\Upsilon}_{j} W & \mathbf{0} & Y_{2 j} & (\star) \\
-\hat{\boldsymbol{\Gamma}}_{j} \mathbf{G}_{1} & G_{2}-\hat{\Upsilon}_{j} W & \mathbf{0} & 0 & 1
\end{array}\right] \succeq 0
$$

are feasible and there exist positive scalars $\beta_{1}$ and $\beta_{2}$ such that for $j=1, \ldots, \mathcal{M}$ the LMIs

$$
\left[\begin{array}{cc}
\beta_{1} & \mathbf{f}^{\mathrm{T}} \\
\mathbf{f} & \mathbf{Y}_{1 j}
\end{array}\right] \succeq 0,\left[\begin{array}{cc}
\beta_{2} & g \\
g & Y_{2 j}
\end{array}\right] \succeq 0
$$

hold. Then the gain $K_{3}$ of the correction term (17) is given by

$$
K_{3}=\frac{W}{G_{2}}
$$

The proof follows the arguments of $[7,5]$, where a parameter independent Lyapunov function was used. 
The following minimization procedure can be applied to this last result:

$$
\begin{gathered}
\min \left(\beta_{1}+\beta_{2}\right), \text { subject to: } \\
\operatorname{diag}\left(\mathbf{Y}_{1 j}, Y_{2 j}\right) \succ 0,(32) \text { and (33) }
\end{gathered}
$$

for $j=1, \ldots, \mathcal{M}$. If a solution exists, $K_{3}$ in (17) is given by (34).

\section{Experimental validation}

\subsection{Equipment details and control design}

The robust ILC design developed in the previous section has been applied experimentally to position tracking of a PMSM (drive A) mechanically connected to a second PMSM (drive B), which is used to generate an external disturbance in the form of a load torque. Between these two motors, an additional rotary mass can be inserted to evaluate the robustness of the design to changes in the mass moment of inertia. The angular position of the motor shaft is measured by a resolver and the resolver to digital converters, used in both drives, which have a resolution of $2 \pi / 2^{14} \mathrm{rad}$ and an accuracy of $\pm 0.007 \mathrm{rad}$. The angular velocity is calculated numerically as a sixteen-point moving average of the position difference with sampling time of the current control loop. The underlying control loops of both PMSMs are based on a field-oriented control approach. This corresponds to decoupling of the orthogonal current components and nonlinear feedback linearization. A detailed description of the experimental setup is given in [5].

The dynamic model for the control of the angular position is

$$
\begin{aligned}
T_{\mathrm{e}}^{\mathrm{A}}(t) & =i_{\mathrm{q}}^{\mathrm{refA}}(t) k_{\mathrm{t}}^{\mathrm{A}} \\
& =J_{\mathrm{M}} \frac{\mathrm{d}^{2} \theta_{\mathrm{m}}^{\mathrm{A}}(t)}{\mathrm{d} t^{2}}+B_{\mathrm{M}} \frac{\mathrm{d} \theta_{\mathrm{m}}^{\mathrm{A}}(t)}{\mathrm{d} t}+T_{\mathrm{l}}^{\mathrm{B}}(t),
\end{aligned}
$$

where $T_{\mathrm{e}}^{\mathrm{A}}$ denotes the electromagnetic torque generated by drive $\mathrm{A}, i_{\mathrm{q}}^{\text {refA }}$ the reference motor current of drive $\mathrm{A}, k_{\mathrm{t}}^{\mathrm{A}}$ the corresponding torque constant, $J_{\mathrm{M}}$ the overall mass moment of inertia, $B_{\mathrm{M}}$ the resulting friction coefficient, $\theta_{\mathrm{m}}^{\mathrm{A}}$ the motor shaft angle, and $T_{1}^{\mathrm{B}}$ the load torque generated by drive $\mathrm{B}$. The resulting continuous-time state-space model for $T_{1}^{\mathrm{B}}=0$ is

$$
\begin{aligned}
& \dot{x}(t)=\left[\begin{array}{cc}
0 & 1 \\
0 & -\frac{B_{\mathrm{M}}}{J_{\mathrm{M}}}
\end{array}\right] x(t)+\left[\begin{array}{c}
0 \\
\frac{k_{\mathrm{t}}^{\mathrm{A}}}{J_{\mathrm{M}}}
\end{array}\right] u(t), \\
& y(t)=\left[\begin{array}{ll}
1 & 0
\end{array}\right] x(t),
\end{aligned}
$$

where

$$
u(t)=i_{\mathrm{q}}^{\mathrm{refA}}(t), x(t)=\left[\begin{array}{c}
\theta_{\mathrm{m}}^{\mathrm{A}}(t) \\
\omega_{\mathrm{m}}^{\mathrm{A}}(t)
\end{array}\right]
$$




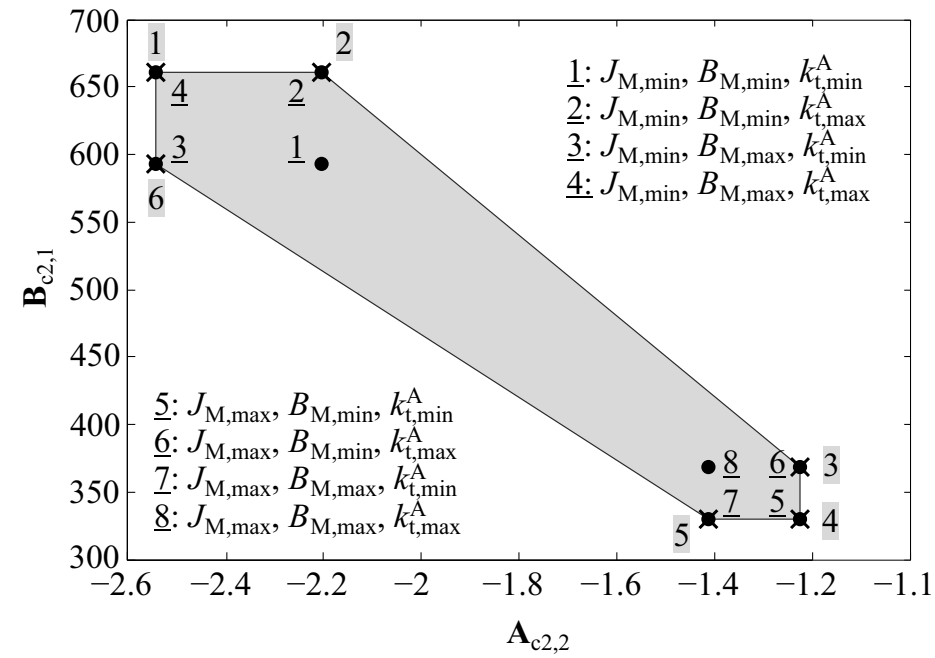

Figure 2: Vertices of the continuous-time plant model (37) marked on the plane formed by the element 2,2 of the state matrix $\mathbf{A}_{\mathrm{c}}$ and the element 2,1 of the input vector $\mathbf{B}_{\mathrm{c}}$.

In this representation, the angular velocity of the motor shaft is $\omega_{\mathrm{m}}^{\mathrm{A}}(t)=\frac{\mathrm{d} \theta_{\mathrm{m}}^{\mathrm{A}}(t)}{\mathrm{d} t}$.

In the remainder of this paper, the model uncertainty is:

- The mass moment of inertia varies between a minimum value $J_{\mathrm{M} \text {,min }}=$ $5.9 \cdot 10^{-4} \mathrm{~kg} \cdot \mathrm{m}^{2}$ and a maximum $J_{\mathrm{M}, \max }=10.6 \cdot 10^{-4} \mathrm{~kg} \cdot \mathrm{m}^{2}$.

- The friction coefficient varies between a minimum value $B_{\mathrm{M}, \min }=1.3$. $10^{-3} \mathrm{~kg} \cdot \mathrm{m}^{2} / \mathrm{s}$ and a maximum $B_{\mathrm{M}, \max }=1.5 \cdot 10^{-3} \mathrm{~kg} \cdot \mathrm{m}^{2} / \mathrm{s}$.

- The torque constant varies between a minimum value $k_{\mathrm{t}, \min }^{\mathrm{A}}=0.35 \mathrm{~N}$. $\mathrm{m} / \mathrm{A}$ and a maximum $k_{\mathrm{t}, \max }^{\mathrm{A}}=0.39 \mathrm{~N} \cdot \mathrm{m} / \mathrm{A}$.

Given these bounds on the parameters in (37), a set of 8 vertices marked by dots in Fig. 2 can be constructed. A minimal convex set containing these vertices consists of the 6 vertices marked by x. Alternatively, MATLAB compatible geometric bounding software can be used to find the 6 vertices of a minimum convex set.

The new design in this paper requires a discrete-time system representation, which is obtained using the Euler discretization method, which preserves the convexity of the uncertain continuous-time state-space model. For the 6 vertices defining the minimum convex set of the continuous-time model (Fig. 2), where the output of each of the models is defined by $\mathbf{C}=\left[\begin{array}{ll}1 & 0\end{array}\right]$. Each of these discrete models has relative degree $r=2$.

The MATLAB toolboxes CVX and SDPT3 have been used to solve the minimization problem (12). Here, the quadratic cost function is defined by the following choices for the weighting matrices $\mathbf{Q}=\operatorname{diag}\left(1.8 \cdot 10^{4}, 0.1,4\right), \mathrm{R}=0.9$ 
and $\gamma=\left[\begin{array}{lll}0.01 & 0 & 0\end{array}\right]^{\mathrm{T}}$. Since the inequality $\mathbf{Y} \succ 0$ cannot be implemented in CVX, it has been replaced by $\mathbf{Y} \succeq 10^{-10}$. I. The numerical solution of the control problem leads to

$$
\mathbf{K}_{\mathrm{s}}=\left[\begin{array}{lll}
-99.4484 & -0.9270 & 1.3916
\end{array}\right]
$$

and hence, using (7), $\mathbf{K}_{1}$ and $K_{2}$.

The design of both the static and dynamic feedforward controllers is based on the parameters of closed-loop system. As detailed in Sec. 3, this is done for $\mathbf{K}_{1}$ arising from (39) and $K_{2}=0$. Moreover, the average values of the system parameters are used, i.e, $J_{\mathrm{M}}=8.25 \cdot 10^{-4} \mathrm{~kg} \cdot \mathrm{m}^{2}, B_{\mathrm{M}}=1.4 \cdot 10^{-3} \mathrm{~kg} \cdot \mathrm{m}^{2} / \mathrm{s}, k_{\mathrm{t}}^{\mathrm{A}}=$ $0.37 \mathrm{~N} \cdot \mathrm{m} / \mathrm{A})$. Application of Euler's discretization method $\left(T_{\mathrm{sm}}^{\mathrm{A}}=2.5 \cdot 10^{-3}\right.$ s) gives the discretized state-space matrices

$$
\mathbf{A}_{\mathrm{av}}=\left[\begin{array}{ll}
1 & 0.0025 \\
0 & 0.9958
\end{array}\right], \quad \mathbf{B}_{\mathrm{av}}=\left[\begin{array}{c}
0 \\
1.1212
\end{array}\right]
$$

and $\mathbf{C}_{\mathrm{av}}=\left[\begin{array}{ll}1 & 0\end{array}\right]$. The state-space model of the closed-loop feedback system used to design both feedforward controllers has the form (see Fig. 1)

$$
\begin{aligned}
x_{k+1}(p+1)= & \left(\mathbf{A}_{\mathrm{av}}+\mathbf{B}_{\mathrm{av}} \mathbf{K}_{1}\right) x_{k+1}(p) \\
& +\mathbf{B}_{\mathrm{av}} w_{k+1}(p), \\
y_{k+1}(p)= & \mathbf{C}_{\mathrm{av}} x_{k+1}(p) .
\end{aligned}
$$

The DC gain of this system is 0.01005547 and hence $N=99.4484$.

The closed-loop system (40) has no zeros and therefore the dynamic feedforward controller is designed using the perfect tracking algorithm [8], which provides the signal $f(p)$ (see Fig. 1) as

$$
f(p)=\frac{1-0.9564 z^{-1}+0.2352 z^{-2}}{0.0028} y^{\mathrm{ref}}(p+r),
$$

Since the relative degree of the plant models is $r=2$, the ILC law (16) in this case is

$$
v_{k+1}(p)=v_{k}(p)+K_{3} e_{k}(p+2) .
$$

Direct application of (42) to the closed-loop state feedback control system (14) gives the state-space model (19) governing the ILC dynamics. The robust ILC design developed in this paper requires this state-space model to be written as a discrete linear repetitive process described by (25). This transformation is non-linear and therefore the set with vertices (26) can be non-convex due to the presence of powers of the matrix $\mathbf{A}_{\mathrm{cl}}(\lambda)$. MATLAB compatible geometric bounding software has therefore been used to find the 6 vertices of a minimum convex set (see (24)) for this example. The MATLAB toolboxes CVX and SDPT3 were also employed to solve the minimization problem (35), where $\mathbf{f}=$ $\left[\begin{array}{lll}0.01 & 0 & 0\end{array}\right]^{\mathrm{T}}, g=0.1$. The strict inequalities $\operatorname{diag}\left(\mathbf{Y}_{1 j}, Y_{2 j}\right) \succ 0$ of (35) also cannot be implemented in CVX. Therefore, they have been replaced by $\operatorname{diag}\left(\mathbf{Y}_{1 j}, Y_{2 j}\right) \succeq 10^{-10} \cdot \mathbf{I}$ for all $j=1, \ldots, 6$. Completing the ILC design gives $K_{3}=0.6942$. 
Table 1: Variation of the reference signal, the load torque signal and the mass moment of inertia during the robust ILC application.

\begin{tabular}{cccc}
\hline Trials & $y^{\mathrm{ref}}(p)$ & $T_{1}^{\mathrm{B}}(p)$ & $J_{\mathrm{M}}\left[\mathrm{kg} \cdot \mathrm{m}^{2}\right]$ \\
\hline $1-30$ & $y_{1}^{\mathrm{ref}}(p)$ & 0 & $5.9 \cdot 10^{-4}$ \\
$31-60$ & $y_{1}^{\mathrm{ref}}(p)$ & $3 k_{\mathrm{t}}^{\mathrm{A}} \mathbb{1}(p-100)$ & $5.9 \cdot 10^{-4}$ \\
$61-90$ & $y_{1}^{\mathrm{ref}}(p)$ & $3 k_{\mathrm{t}}^{\mathrm{A}} \mathbb{1}(p-100)$ & $10.6 \cdot 10^{-4}$ \\
$91-500$ & $y_{2}^{\mathrm{ref}}(p)$ & $2 k_{\mathrm{t}}^{\mathrm{A}} \mathbb{1}(p-250)$ & $8.6 \cdot 10^{-4}$ \\
\hline
\end{tabular}

\subsection{Experimental results and discussion}

To counteract high frequency noise and quantization errors in the implementation, an off-line low-pass filtering of the tracking error has been implemented at the end of each trial. For this purpose, a zero-phase digital filter, available in MATLAB through the function filtfilt, was used. This filter is parameterized as a 2nd-order low-pass digital Butterworth filter with a cutoff frequency of $30 \mathrm{~Hz}$ and sampling period $T_{\mathrm{sm}}^{\mathrm{A}}$.

In the remainder of this section, the experimental results obtained for four control strategies are given to demonstrate the capabilities of the new ILC design. The following alternatives are investigated and compared to each other:

1. the new ILC structure shown in Fig. 1,

2. a simplified ILC structure where an ILC signal acts only on the plant input - this structure is detailed at the relevant point below,

3. an ILC structure without integral action,

4. a non-ILC structure consisting of a state feedback controller with integral action and a dynamic feedforward controller.

These experiments include the impact of a-priori unknown disturbances and also model uncertainty. The disturbances are applied as variations in the load torque, whereas model uncertainty is addressed by exchanging the mass moment of inertia after a particular number of trials have been executed. Moreover, the effects of changes in the reference trajectory are also investigated. Table 1 and Fig. 3 give the parameters and signals used. In all experiments, the torque constant and the friction coefficient are kept constant.

In the computation of the ILC laws at any sampling instant on the current trial, the tracking error contribution from the previous trial is shifted by $r=2$ samples, see (42). Hence the last two samples of the ILC signal, i.e., $v_{k+1}(999)$ and $v_{k+1}(1000)$ cannot be implemented and instead the following assignments were made $e_{k+1}(1001)=e_{k+1}(1000), e_{k+1}(1002)=e_{k+1}(1000)$. Hence, the first two samples of the tracking error from the previous trial are not used and in each case replaced by a zero. 


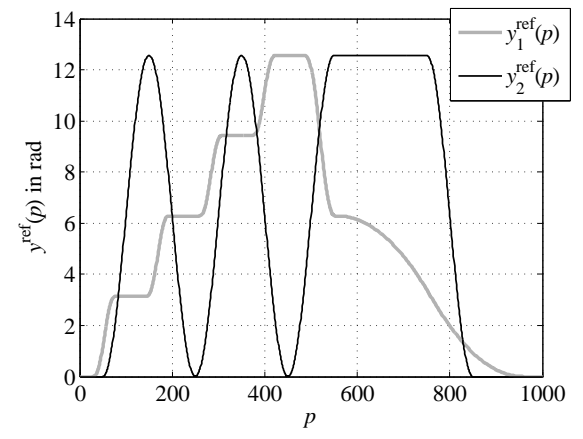

Figure 3: Reference trajectory (a) and the load torque generated by drive B (b) during the robust ILC.

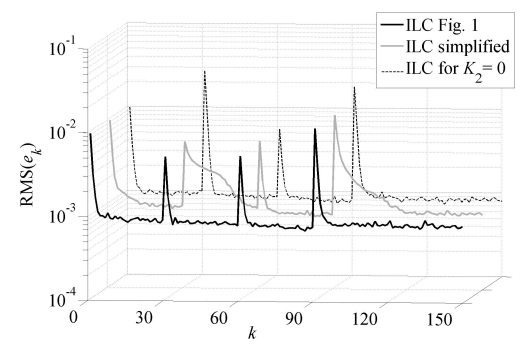

Figure 4: RMS values of the tracking error for the first 150 trials.

Assuming zero boundary conditions, the new ILC scheme shown in Fig. 1 according to the scenario in Table 1 has been experimentally tested. The rootmean-square (RMS) tracking error for experimental data is shown in Fig. 4, where the corresponding plot is denoted by "ILC Fig. 1".

These results show that the new ILC needs 4 trials to result in very small tracking errors that are close to the RDC resolution - despite all the investigated changes in load torque, the mass moment of inertia and the reference trajectory. Moreover, the integral action of the state feedback controller reduced the steady-state tracking error caused by the disturbance torque starting immediately during the trial in which the disturbance appeared.

Evaluation of a simplified ILC structure. A simplification of Fig. 1 occurs when where the ILC signal $v_{k+1}(p)$ is connected only to the system input. An identical set of experiments were performed for this alternative scheme. For the first 150 executed trials, the resulting RMS tracking error is denoted by "ILC Fig. 9" in Fig. 4. For this simplified ILC structure, slower trial-to-trial error convergence occur after adding or changing load disturbances, where 14 trials, as opposed to only 4, are required to obtain tracking errors close to the $\mathrm{RDC}$ resolution. This effect is related to changes in the integral action output signal $\psi_{k+1}(p)$ until perfect tracking is obtained again. 
Evaluation of the ILC structure without integral action. The ILC schemes considered above are equivalent for $K_{2}=0$, i.e., when the integral action is disabled. A set of experiments was also performed for this case. The resulting RMS tracking error is denoted by "ILC for $K_{2}=0$ and shown in Fig. 4". This set of experiments is of particular interest in assessing the tracking error after adding a load torque, on trial $k=31$. In this case, a steady-state tracking error occurs that can only be reduced/counteracted by the ILC after the next trial has begun. The RMS tracking error is significantly larger at trial $k=31$ than in both previous sets of experiments, justifying the inclusion of the integral action.

\section{Conclusions}

In this paper, an innovative ILC law, augmented by a state feedback controller with integral action, has been developed and applied to the angular position tracking of a PMSM. The integral action included in the state feedback controller reduces the steady-state error caused by a load disturbance torque during the trial in which it appears. The new robust ILC design does not require the storage of the additional signal which is the product of the gain vector and the state vector along the trials. It can be used for plants with relative degree $r \geq 1$ and also in the case of uncertainty in the plant model parameters. The results confirm that the new ILC is capable of achieving small tracking errors after a small number of trials (4 trials for the considered PMSM), including the case

when model uncertainty, unmeasured disturbances and measurement noise are present.

Given the results and the positive experimental validation reported in this paper, there are a number of areas for future research. These include implementation on more sophisticated drive train topologies, e.g., those involving elasticity. Also ILC design in the presence of noise should be investigated. The analysis and experimental results in this paper are for SISO systems, Extension of the theory to case of square multi-input multi-output systems should be considered. In the PMSM application the state vector entries can be directly measured. If in other applications this is not the case then an observer is required and this is another area for further research, including dispensing with state feedback.

\section{References}

[1] S. Arimoto, S. Kawamura, F. Miyazaki, Bettering operation of robots by learning, Journal of Robotic Systems 1 (2) (1984) 123-140. doi:10.1002/rob.4620010203.

[2] H.-S. Ahn, Y.-Q. Chen, K. L. Moore, Iterative learning control: Brief survey and categorization, IEEE Transactions on Systems, Man and Cybernetics, Part C 37 (6) (2007) 1099-1121. doi:10.1109/TSMCC.2007.905759. 
[3] D. A. Bristow, M. Tharayil, A. G. Alleyne, A survey of iterative learning control, IEEE Control Systems Magazine 26 (3) (2006) 96-114. doi:10.1109/MCS.2006.1636313.

[4] W. Paszke, E. Rogers, K. Galkowski, Experimentally verified generalized KYP lemma based iterative learning control design, Control Engineering Practice 53 (2016) 57 - 67. doi:10.1016/j.conengprac.2016.04.011.

[5] S. Mandra, K. Galkowski, H. Aschemann, Robust guaranteed cost ILC with dynamic feedforward and disturbance compensation for accurate PMSM position control, Control Engineering Practice 65 (2017) 36 - 47. doi:10.1016/j.conengprac.2017.05.004.

[6] I. R. Petersen, D. C. McFarlane, Optimal guaranteed cost control and filtering for uncertain linear systems, IEEE Transactions on Automatic Control 39 (9) (1994) 1971-1977. doi:10.1109/9.317138.

[7] E. Rogers, K. Galkowski, D. H. Owens, Control systems theory and applications for linear repetitive processes, Vol. 349 of Lecture Notes in Control and Information Sciences, Springer-Verlag, Berlin, Germany, 2007.

[8] M. Tomizuka, Zero phase error tracking algorithm for digital control, Journal of Dynamic Systems, Measurement, and Control 109 (1) (1987) 65-68. doi: $10.1115 / 1.3143822$. 